\title{
Subcortical stimulation mapping of descending motor pathways for perirolandic gliomas: assessment of morbidity and functional outcome in $\mathbf{7 0 2}$ cases
}

\author{
Seunggu J. Han, MD, ${ }^{1,2}$ Ramin A. Morshed, MD, ${ }^{1}$ Irene Troncon, MD, ${ }^{3}$ Kesshi M. Jordan, PhD, ${ }^{4}$ \\ Roland G. Henry, PhD, ${ }^{4}$ Shawn L. Hervey-Jumper, MD, ${ }^{1}$ and Mitchel S. Berger, MD1 \\ 'Department of Neurological Surgery, University of California, San Francisco, California; '2Department of Neurological Surgery, \\ Oregon Health and Science University, Portland, Oregon; ${ }^{3}$ Department of Neurological Surgery, Padua University Hospital, \\ Padua, Italy; and ${ }^{4}$ Department of Neurology, University of California, San Francisco, California
}

\begin{abstract}
OBJECTIVE Herein, the authors report their experience with intraoperative stimulation mapping to locate the descending subcortical motor pathways in patients undergoing surgery for hemispheric gliomas within or adjacent to the rolandic cortex, with particular description of the morbidity and functional outcomes associated with this technique.

METHODS This is a retrospective analysis of patients who, in the period between 1997 and 2016, had undergone resection of hemispheric perirolandic gliomas within or adjacent to descending motor pathways. Data regarding intraoperative stimulation mapping and patient postoperative neurological status were collected.

RESULTS Of 702 patients, stimulation mapping identified the descending motor pathways in 300 cases (43\%). A new or worsened motor deficit was seen postoperatively in 210 cases (30\%). Among these 210 cases, there was improvement in motor function to baseline levels by 3 months postoperatively in 161 cases (77\%), whereas the deficit remained in 49 cases $(23 \%)$. The majority (65\%) of long-term deficits (persisting beyond 3 months) were mild or moderate (antigravity strength or better). On multivariate analysis, patients in whom the subcortical motor pathways had been identified with stimulation mapping during surgery were more likely to develop an additional and/or worsened motor deficit postoperatively than were those in whom the subcortical pathways had not been found ( $45 \%$ vs $19 \%$, respectively, $p<0.001)$. This difference remained when considering the likelihood of a long-term deficit (i.e., persisting $>3$ months; $12 \%$ vs $3.2 \%$, $p$ $<0.001)$. A higher tumor grade and the presence of a preoperative motor deficit were also associated with higher rates of motor deficits persisting long-term. A region of restricted diffusion adjacent to the resection cavity was seen in 20 patients with long-term deficits (41\%) and was more common in cases in which the motor pathways were not identified (69\%). Long-term deficits that occur in settings in which the subcortical motor pathways are not identified seem in large part due to ischemic injury to descending tracts.
\end{abstract}

CONCLUSIONS Stimulation mapping allows surgeons to identify the descending motor pathways during resection of tumors in perirolandic regions and to attain an acceptable rate of morbidity in these high-risk cases.

https://thejns.org/doi/abs/10.3171/2018.3.JNS172494

KEYWORDS motor mapping; stimulation mapping; subcortical mapping; oncology

$\mathrm{G}$ LIAL tumors involving the motor cortex or descending subcortical motor fibers present a unique set of challenges. With the main objective of achieving maximal resection with minimal morbidity, the neurosurgeon's ability to safely and reliably identify and preserve the motor cortex as well as its associated descending white matter tracts is a crucial step in surgical management. ${ }^{4,12,17}$ The utility of stimulation mapping to localize and confirm eloquent structures and minimize neurological morbidity during resection of gliomas has been well established and adopted as the standard of care at many centers. ${ }^{10}$ Advances in preoperative imaging modalities, including positron emission tomography, functional MRI, magnetoencephalography, and diffusion MRI fiber tracking (DMRI-FT), offer additional methods to facilitate identification of functional brain in relationship to tumor. ${ }^{29}$ Information gath-

ABBREVIATIONS CST = corticospinal tract; DMRI-FT = diffusion MRI fiber tracking; DWI = diffusion weighted imaging.

SUBMITTED October 2, 2017. ACCEPTED March 19, 2018.

INCLUDE WHEN CITING Published online August 17, 2018; DOI: 10.3171/2018.3.JNS172494. 
ered with these modalities can also be loaded into neuronavigational systems to help localize functional tissue. In the case of DMRI-FT, the path of white matter fibers such as the corticospinal tract (CST) can be modeled and displayed by the navigation system in 3D space. ${ }^{34}$ However, these techniques are still susceptible to the consequences of brain shift and loss of navigational accuracy. ${ }^{20}$ In addition, reconstruction of motor fibers using DMRI-FT is highly user and technique dependent, and the depicted fibers may not be an accurate representation of the true functional fibers. ${ }^{1,18} \mathrm{~A}$ false-negative absence of DMRI-FT streamlines can occur in edematous or tumor-infiltrating regions, ${ }^{2,6}$ or false-positive streamline bundles can be seen when pathways are displaced by growth. Intraoperative MRI can address some of these challenges but requires significant added time and resources, and evidence supporting a robust correlation with intraoperative functional mapping data is currently lacking. ${ }^{22,24}$ Given the current state of technology, we have continued to rely on stimulation mapping as the gold-standard for localizing functional tissue. In this paper, we report our experience in using subcortical motor mapping to localize and preserve descending motor fibers during tumor resection. We describe the utility of this technique in a high-risk cohort of patients harboring gliomas involving the motor cortex or its descending fibers as well as the short- and long-term functional outcomes of the patients. Having previously described the use of subcortical mapping during glioma surgery, ${ }^{10}$ we present this follow-up analysis of just over 700 cases, showing that this technique continues to be reliable and robust and reduces injury to the subcortical motor tracts during glioma surgery.

\section{Methods}

\section{Patient Selection and Characteristics}

The study cohort includes all patients with hemispheric gliomas within or adjacent to the rolandic cortex and descending motor fibers who underwent resection during which cortical and subcortical stimulation mapping was used. All patients were treated at the University of California, San Francisco Medical Center by the senior author (M.S.B.) between 1997 and 2016. Patients were initially identified by querying a prospectively collected brain tumor registry, which contained findings related to intraoperative cortical and subcortical mapping results. Additional data regarding the patients' baseline demographic and clinical characteristics, tumor histology, and pre- and postoperative neurological status were collected through a review of the medical center inpatient and outpatient records, operative reports, and pathology reports. All patients with a new or worsened postoperative motor deficit were followed up for at least 3 months postoperatively, and, for purposes of the functional outcome evaluation, the deficit was considered long-term if it remained at 3 months. The study was conducted with the approval of the local Committee on Human Research.

\section{Mapping Technique}

The technique for subcortical mapping has been described in prior reports. ${ }^{4,11,13,17}$ The Ojemann stimulator
(Radionics, Inc.) was used with a bipolar probe with 5-mm spacing between electrodes to deliver a biphasic squarewave pulse of 1-msec duration at $60 \mathrm{~Hz}$. For awake patients, visualized motor response with verbal confirmation from the patient was used to determine a positive motor response, and multichannel electromyography recordings were used in asleep patients. ${ }^{13,35}$ Following dural opening, the cortical motor region was stimulated and identified using serially increasing currents in increments of $1 \mathrm{~mA}$, starting at $2 \mathrm{~mA}$ for awake patients (to maximum of $6 \mathrm{~mA}$ ) and at $4 \mathrm{~mA}$ for asleep patients (to maximum of $16 \mathrm{~mA}$ ). Then the same current applied at the cortex was used for the subcortical mapping to localize the descending motor pathways. Subcortical stimulation mapping was started when the resection was carried to the depth on level with the bottom of a sulcus. Serial subcortical stimulation was performed with each advancing 2-3 $\mathrm{mm}$ of resection. When cortical motor sites could not be identified due to safety of access (e.g., from scar tissue preventing adequate exposure), subcortical mapping was still performed in an attempt to identify the motor pathways using a current between 10 and $16 \mathrm{~mA}$ for asleep patients and between 4 and $6 \mathrm{~mA}$ for awake patients.

\section{Imaging}

For patients who developed a postoperative deficit, the early postoperative MRI (within 72 hours) was reviewed for any regions of abnormality on diffusion-weighted imaging (DWI). For our analysis, the degree of abnormality was arbitrarily considered significant when the area of restricted diffusion along the resection cavity was greater than $2 \mathrm{~mm}$ in thickness. Patients in the two illustrative cases underwent a high angular resolution diffusion imaging (HARDI) sequence on a 3-T General Electric Medical Systems scanner (Discovery MR750) both during the presurgical MRI and the early postoperative MRI with the following parameters: TR $6425 \mathrm{msec}$, TE $80 \mathrm{msec}$, axial slices 50, in-plane $2.2 \mathrm{~mm}^{2}$ (subsampled to $1.1 \mathrm{~mm}^{2}$ ), slice thickness $2 \mathrm{~mm}$, b value $2000 \mathrm{sec} / \mathrm{mm}^{2}$, diffusion gradients 55 , minimally diffusion-weighted image 1 .

\section{Illustrative Cases}

Two illustrative cases were selected to explore damage to the CST by modeling the pathway using tractography (Fig. 1). The first case was a 62-year-old female with a recurrent right frontal anaplastic astrocytoma who underwent repeat resection after presenting with worsening left-sided weakness and headaches. The repeat resection revealed glioblastoma on pathological analysis. The patient in this case suffered a long-term deficit, but the subcortical pathway was not identified by stimulation. The second case was a 38-year-old male with a recurrent left parietal low-grade astrocytoma who had new, worsening enhancement on imaging concerning for a high-grade recurrence. Repeat resection was undertaken, and pathological analysis demonstrated anaplastic astrocytoma. In this case, the subcortical pathway was identified, and the patient went on to suffer a long-term deficit. In each of these illustrative cases, the presurgical CST was modeled with residual bootstrap Q-ball probabilistic tractography using 

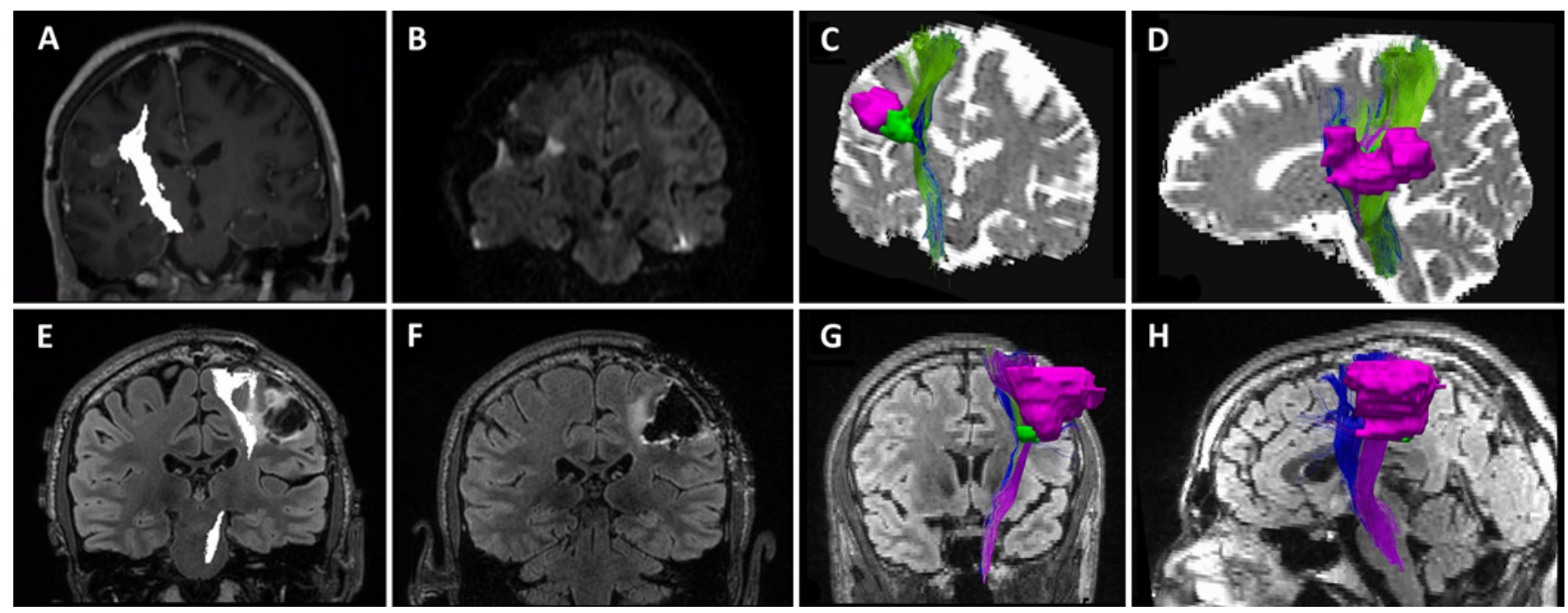

FIG. 1. Two illustrative cases used to explore mechanisms of injury to the descending motor pathways. To explore possible motor tract injury due to mechanical and/or ischemic injury, two cases were selected from a cohort of patients who had suffered a longterm deficit. Preoperative images ( $\mathbf{A}$ and $\mathbf{E})$ demonstrate the relationship of the modeled fibers to the tumor, and postoperative images ( $\mathbf{B}$ and $\mathbf{F}$ ) demonstrate the resection cavity and the region of restricted diffusion. The technique of motor fiber modeling is described in Methods. The volume of tissue with restricted diffusion (green) and the resection cavity (pink) on postoperative images were manually segmented (C, D, G, and H). Portions of the DMRI-FT motor fibers (blue) overlapping with the resection cavity are displayed in pink, and portions overlapping with the region of restricted diffusion are shown in green. In the first case (A-D), the patient suffered a long-term deficit, but the subcortical fibers were never identified by stimulation. Interestingly, the DMRI-FT CST did not overlap with the resection cavity, but a large portion of the motor fibers is seen running through the region of postoperative restricted DWI. In contrast, in the second case $(\mathrm{E}-\mathrm{H})$, motor tracts were identified by subcortical mapping during surgery, and the patient experienced persistent motor deficits. As expected, a significant portion of the descending motor fibers is seen running through the resection cavity. Figure is available in color online only.

the preprocessing parameters previously described..$^{5,18}$ The region of restricted diffusion and the resection cavity on postoperative images were manually segmented in TrackVis (trackvis.org). A rigid registration was performed with FLIRT (FMRIB's Linear Image Registration Tool, FSL) to bring the restricted diffusion and surgical cavity regions of interest into the preoperative space so that the preoperative CST could be targeted to infer potential damage attributed to resection (pink) and restricted diffusion (green). ${ }^{14,15}$

\section{Statistical Analysis}

Comparisons of the likelihood of developing any or long-term deficits postoperatively between groups were performed using the chi-square test or Fisher's exact test. Multivariate analysis was performed using a logistic regression analysis (JMP 13, SAS Institute Inc.). A p value < 0.05 was considered significant for all analyses.

\section{Results}

Table 1 depicts demographics of the surgical cohort in addition to rates of new or worsened and long-term postoperative deficits. The age of the patients ranged from 18 to 84 years (median 44 years). There were 276 patients with low-grade tumors (WHO grade I or II) and 426 patients with high-grade tumors (WHO grade III or IV). One hundred ninety-two patients (28\%) underwent an awake mapping procedure. A motor deficit was present in 89 patients $(13 \%)$ prior to surgery. Subcortical stimulation mapping was performed in all patients, and subcortical motor tracts were successfully identified in 300 cases (43\%). A total of 210 patients $(30 \%)$ developed a new or worsened motor deficit within 24 hours after surgery. Of these patients, 161 (77\%) recovered to normal or their preoperative baseline function by the 3 rd postoperative month. The remaining 49 patients, representing $7.0 \%$ of the entire cohort, were considered to have a long-term deficit.

Patients in whom the subcortical motor pathways were localized by stimulation mapping were more likely to develop a new or worsened motor deficit postoperatively than those in whom the subcortical mapping did not identify the pathway (45\% vs $19 \%$, respectively, $\mathrm{p}<0.001$ ). When only long-term motor deficits were considered, the group in which the motor pathways had been identified continued to be associated with higher rates of postoperative deficits ( $12 \%$ vs $3.2 \%$, respectively; $\mathrm{p}<0.001)$. $\mathrm{Pa}-$ tients who had an existing baseline motor deficit were also more likely to develop a new or worsened deficit than were those who did not have a preoperative deficit ( $65 \%$ vs $25 \%$, respectively; $p<0.001)$. This difference remained when considering only long-term deficits $(16 \%$ for those with preoperative deficits vs $5.7 \%$ for those without; $p=0.003$ ). A frontal lobe location was also associated with significantly higher rates of postoperative deficits. On univariate and multivariate analysis, tumor location, preoperative motor deficit, and identification of the subcortical pathway intraoperatively were significantly associated with a new or worsened postoperative neurological deficit. However, only identification of the subcortical pathway intraop- 
TABLE 1. Patient and tumor characteristics and univariate analysis of those who underwent resection of perirolandic gliomas

\begin{tabular}{|c|c|c|c|c|c|}
\hline Parameter & Total & New/Worse Deficit & $p$ Value & Long-Term Deficit & $p$ Value \\
\hline No. of patients & 702 & $210(30 \%)$ & & $49(7.0 \%)$ & \\
\hline Mean age (yrs) & $44.9 \pm 13.5$ & * & & $\dagger$ & \\
\hline Sex & & & 0.62 & & 0.93 \\
\hline Male & $411(58.5 \%)$ & $120(29.2 \%)$ & & $29(7.1 \%)$ & \\
\hline Female & $291(41.5 \%)$ & $90(30.9 \%)$ & & $20(6.9 \%)$ & \\
\hline Side & & & 0.88 & & 0.50 \\
\hline Lt & $302(43.0 \%)$ & $89(29.5 \%)$ & & $20(6.6 \%)$ & \\
\hline Rt & $398(56.7 \%)$ & $119(29.9 \%)$ & & $28(7.0 \%)$ & \\
\hline Bilat & $2(0.3 \%)$ & $2(100 \%)$ & & $1(50 \%)$ & \\
\hline Tumor location & & & $<0.001$ & & 0.25 \\
\hline Frontal & $279(39.7 \%)$ & $110(39.4 \%)$ & & $24(8.6 \%)$ & \\
\hline Temporal & $73(10.4 \%)$ & $10(13.7 \%)$ & & $2(2.7 \%)$ & \\
\hline Parietal & $131(18.7 \%)$ & $37(28.2 \%)$ & & $10(7.6 \%)$ & \\
\hline Insular & $94(13.4 \%)$ & $14(14.9 \%)$ & & $3(3.2 \%)$ & \\
\hline Multilobular & $125(17.8 \%)$ & $39(31.2 \%)$ & & $10(8.0 \%)$ & \\
\hline WHO grade & & & 0.47 & & 0.17 \\
\hline I & $4(0.6 \%)$ & $0(0 \%)$ & & $0(0 \%)$ & \\
\hline$\|$ & $272(38.7 \%)$ & $85(31.3 \%)$ & & $12(4.4 \%)$ & \\
\hline III & $216(30.7 \%)$ & $60(27.8 \%)$ & & $18(8.3 \%)$ & \\
\hline IV & $210(30.0 \%)$ & $65(31.0 \%)$ & & $19(9.0 \%)$ & \\
\hline Anesthesiaf & & & 0.76 & & 0.18 \\
\hline Asleep & $484(71.6 \%)$ & $152(31.4 \%)$ & & $31(6.4 \%)$ & \\
\hline Awake & $192(28 \%)$ & $58(30.2 \%)$ & & $18(9.4 \%)$ & \\
\hline Preop motor deficit & & & $<0.001$ & & $<0.001$ \\
\hline Yes & $89(12.7 \%)$ & $58(65 \%)$ & & $14(16 \%)$ & \\
\hline No & $613(87.3 \%)$ & $152(25 \%)$ & & $35(5.7 \%)$ & \\
\hline Subcortical pathway found & & & $<0.001$ & & $<0.001$ \\
\hline Yes & $300(42.7 \%)$ & $135(45 \%)$ & & $36(12 \%)$ & \\
\hline No & $402(57.3 \%)$ & $75(19 \%)$ & & $13(3.2 \%)$ & \\
\hline
\end{tabular}

* OR $=1.00,95 \% \mathrm{Cl} 0.99-1.02$.

† OR $=1.01,95 \% \mathrm{Cl}$ 0.99-1.04.

$\ddagger$ Data for 26 patients unavailable.

eratively was associated with long-term motor deficits on both univariate and multivariate analysis (Table 2).

Among the 49 patients with persistent long-term deficits, 30 had a mild deficit, i.e., 4/5 strength or better or isolated facial weakness. In 2 patients the deficit was moderate (3/5, antigravity strength), and in 17 patients the effects were severe (unable to move against gravity). Tables 3 and 4 describe the severity of deficits and the distribution of affected body groups among the patients with long-term deficits.

Significant regions of DWI restriction were seen on the immediate postoperative scan in 20 (41\%) of the 49 patients with long-term deficits. Among the 13 cases in which the subcortical pathway was never identified with stimulation yet the patients went on to develop a long-term deficit, 9 patients $(69 \%)$ had DWI restricted regions on the postoperative scans. The presence of this DWI abnormality was significantly more common among the patients in whom subcortical pathways were not identified and went on to have a long-term deficit, as compared to those in whom subcortical pathways were found and a long-term deficit occurred (69\% vs 31\%, respectively; Table 5).

\section{Discussion}

Diffuse gliomas, by nature, invade and infiltrate white matter tracts, and injury to functional subcortical pathways can be responsible for a large portion of morbidity encountered when surgically removing tumors near functional tissue..$^{8,26,30}$ Safe resection of tumors near functional areas necessitates intraoperative identification of functional sites both at the cortical and the subcortical levels. Techniques for subcortical stimulation mapping using biphasic bipolar 60-Hz stimuli for glioma surgery have been described by our group and others.,11,12,17 More recently, monopolar high-frequency stimulation parameters have been increasingly used and studied, taking advantage of the more predictable pattern of current spread. In theory, the amount of current needed to stimulate a white matter tract can be used to estimate the distance to the stimulated tract. ${ }^{3,23}$ A number of studies have evaluated the relationship of stimulation intensity with the distance between the stimulation points and the motor fibers modeled by using DMRI-FT. 2,5,19,23,31,36 There is considerable variability in the reported relationships between the current intensity 
TABLE 2. Multivariate analysis

\begin{tabular}{|c|c|c|c|c|c|}
\hline Variable & Total & New/Worse Deficit, OR (95\% Cl) & p Value & Long-Term Deficit, OR (95\% Cl) & $\mathrm{p}$ Value \\
\hline \multicolumn{6}{|l|}{ Tumor location } \\
\hline Frontal & $279(39.7 \%)$ & 1 & & & \\
\hline Temporal & $73(10.4 \%)$ & $0.23(0.11-0.49)$ & $<0.001$ & & \\
\hline Parietal & $131(18.7 \%)$ & $0.55(0.34-0.89)$ & 0.013 & & \\
\hline Insular & $94(13.4 \%)$ & $0.43(0.23-0.81)$ & 0.006 & & \\
\hline Multilobular & $125(17.8 \%)$ & $0.72(0.45-1.17)$ & 0.18 & & \\
\hline \multicolumn{6}{|l|}{ WHO grade } \\
\hline I & $4(0.6 \%)$ & & & NA & NA \\
\hline$\|$ & $272(38.7 \%)$ & & & 1 & \\
\hline III & $216(30.7 \%)$ & & & $2.17(0.99-4.74)$ & 0.051 \\
\hline IV & $210(30.0 \%)$ & & & $2.29(0.99-5.33)$ & 0.054 \\
\hline \multicolumn{6}{|l|}{ Anesthesia } \\
\hline Asleep & $484(71.6 \%)$ & & & 1 & \\
\hline Awake & $192(28 \%)$ & & & $1.67(0.86-3.25)$ & 0.13 \\
\hline \multicolumn{6}{|l|}{ Preop motor deficit } \\
\hline Yes & $89(12.7 \%)$ & $3.16(1.95-5.17)$ & $<0.001$ & $2.10(0.99-4.42)$ & 0.052 \\
\hline No & $613(87.3 \%)$ & 1 & & 1 & \\
\hline \multicolumn{6}{|c|}{ Subcortical pathway found } \\
\hline Yes & $300(42.7 \%)$ & $3.17(2.23-4.54)$ & $<0.001$ & $7.94(3.52-17.4)$ & $<0.001$ \\
\hline No & $402(57.3 \%)$ & 1 & & 1 & \\
\hline
\end{tabular}

$\mathrm{NA}=$ not applicable.

and the measured distance to the DMRI-FT motor fibers; some authors have proposed a linear relationship of $1 \mathrm{~mA}$ of current being able to stimulate structures $1 \mathrm{~mm}$ away, while others have shown that this relation is not strictly linear. ${ }^{16,21,24}$ Some of this variability may be due to differences in techniques, both in the stimulation protocol (e.g., probe type, stimulation parameters) and the DMRIFT methodologies, which have well-documented reproducibility concerns (e.g., operator dependence, sequence, tractography algorithm, parameter choice, etc.). ${ }^{9,32,33}$ In addition, these studies tend to estimate distances to reconstructed fiber tracts based on preoperative or postoperative DMRI studies, which are susceptible to brain shift and loss of navigational accuracy.
Nonetheless, the overarching theme remains that positive subcortical motor stimulation at low currents likely suggests that the motor pathways are close, while stimulation requiring higher currents indicates greater distance to those functional pathways. When considering the impact on functional outcomes, there is also general consensus in the literature that the resection should be stopped at a safe margin distance from the descending motor pathways. This margin translated into stimulation threshold ranges from 1 to $10 \mathrm{~mA}$, and resection to positive stimulation levels below these currents has resulted in significantly higher rates of postoperative motor deficits. ${ }^{16,21,23,24,27,28} \mathrm{At}$ our institution, the Penfield technique of bipolar stimulation mapping has been used with these parameters for

TABLE 3. Incidence of additional postoperative motor deficits

\begin{tabular}{|c|c|c|c|c|c|}
\hline \multirow[b]{2}{*}{ Parameter } & \multicolumn{4}{|c|}{ Long-Term Deficit } & \multirow[b]{2}{*}{$\begin{array}{c}p \\
\text { Value }\end{array}$} \\
\hline & Total No. & $\begin{array}{c}\text { Mild } \\
\text { (4/5 strength or better or isolated face weakness) }\end{array}$ & $\begin{array}{c}\text { Moderate } \\
\text { (3/5 strength) }\end{array}$ & $\begin{array}{c}\text { Severe } \\
\text { (2/5 strength or worse) }\end{array}$ & \\
\hline All patients & $49(7.0 \%)$ & $30(4.3 \%)$ & $2(0.3 \%)$ & $17(2.4 \%)$ & \\
\hline Subcortical pathway & & & & & $<0.001$ \\
\hline Found & $36(12 \%)$ & $23(7.7 \%)$ & $1(0.3 \%)$ & $12(4 \%)$ & \\
\hline Not found & $13(3.2 \%)$ & $7(1.7 \%)$ & $1(0.2 \%)$ & $5(1.2 \%)$ & \\
\hline Histology & & & & & 0.03 \\
\hline High grade & $37(8.7 \%)$ & $20(4.7 \%)$ & $0(0 \%)$ & $17(4.0 \%)$ & \\
\hline Low grade & $12(4.4 \%)$ & $10(3.6 \%)$ & $2(0.7 \%)$ & $0(0 \%)$ & \\
\hline Preop motor deficit & & & & & 0.003 \\
\hline Yes & $14(16 \%)$ & $6(6.7 \%)$ & $0(0 \%)$ & $8(9.0 \%)$ & \\
\hline No & $35(5.7 \%)$ & $24(3.9 \%)$ & $2(0.3 \%)$ & $9(1.5 \%)$ & \\
\hline
\end{tabular}


TABLE 4. Types of long-term motor deficits

\begin{tabular}{lcccccc}
\hline & \multicolumn{1}{c}{ No. of Patients } \\
\cline { 2 - 7 } \multicolumn{1}{c}{ Effect } & Total & Face & $\begin{array}{c}\text { Upper } \\
\text { Extremity }\end{array}$ & $\begin{array}{c}\text { Lower } \\
\text { Extremity }\end{array}$ & $\begin{array}{c}\text { Upper \& Lower } \\
\text { Extremity }\end{array}$ & $\begin{array}{c}\text { Significant DWI } \\
\text { Restricted Area }\end{array}$ \\
\hline Mild (4/5 strength or better or isolated face) & 30 & $1(3.3 \%)$ & $8(27 \%)$ & $7(23 \%)$ & $14(47 \%)$ & $9(30 \%)$ \\
\hline Moderate (3/5 strength) & 2 & 0 & $1(50 \%)$ & 0 & $1(50 \%)$ & $1(50 \%)$ \\
\hline Severe (2/5 strength or worse) & 17 & 0 & $2(12 \%)$ & $1(5.9 \%)$ & $14(82 \%)$ & $10(59 \%)$ \\
\hline
\end{tabular}

subcortical stimulation mapping for the last 20 years. This technique is well established in its safety and efficacy, and we have found positive stimulation results to be unambiguous and reliable. Resection is stopped when there is positive stimulation of the descending pathways. We have also found negative results to be informative. According to our prior work, with negative mapping, very low functional morbidity rates (approximately $2 \%$ ) can be expected. ${ }^{17}$

The current analysis expands on our previous report ${ }^{17}$ and describes the utility of stimulation mapping of the descending subcortical motor pathways and its impact on postoperative neurological morbidity in a large cohort of 702 patients with perirolandic gliomas. This report again illustrates the high-risk nature of these particular lesions as we encountered a relatively high rate of functional morbidity in the immediate postoperative period $(30 \%){ }^{7} \mathrm{How}-$ ever, most of these functional morbidities resolved; only a small subset had any motor deficits that persisted longterm (7\%), and only $2.4 \%$ experienced a persistent severe disabling deficit. Our findings also support prior reports of patients with an existing preoperative motor deficit who are at greater risk for worsening neurological function after surgery. ${ }^{17,30}$ Although histological tumor grade was not associated with the likelihood of developing a new or worsened motor deficit after surgery, higher rates of long-term morbidity were found in high-grade tumors. The DMRI-FT studies have revealed that high-grade gliomas tend to more frequently displace and distort the CSTs, whereas low-grade gliomas tend to infiltrate the motor fibers. ${ }^{1}$ Perhaps the greater degree of associated edema and necrosis in high-grade tumors limits the ability of white matter to recover after surgery.

The utility and reliability of subcortical mapping is demonstrated by our findings that intraoperative mapping results could predict the likelihood of developing immediate and permanent motor deficits. When the subcortical motor pathways could be identified intraoperatively, there was a significantly higher risk of developing not only a new postoperative deficit, but also a permanent deficit persisting beyond 3 months, compared to cases in which the motor fibers could not be identified. For long-term deficits, there was an almost fourfold increase in the rate of deficits when the subcortical fibers were encountered by stimulation mapping. This suggests that when the resection was done close to the motor fibers, there was an increased risk of injury to these tracts and subsequent morbidity. In addition, it is possible that transected fibers can still be transiently stimulated to evoke a motor response during subcortical mapping. Thus, stimulation mapping provides valuable feedback on when the resection should be stopped, based on a robust clinical correlation; proceeding without this information could likely result in even higher functional morbidity rates.

Vascular injury to subcortical motor fibers at the time of surgery can also lead to significant deficits that typically do not improve with time..$^{21,25,30}$ In our cohort, significant regions of DWI restriction were observed on the immediate postoperative scan of $41 \%$ of patients who experienced a long-term deficit. Interestingly, in this subcohort experiencing long-term deficits, significant regions of DWI abnormality on postoperative imaging were observed much more frequently among the cases in which the subcortical motor pathways were not found than in the cases in which subcortical pathways were found by stimulation. We believe this reflects the two different major causes of long-term morbidity between the groups: mechanical injury to the tracts and vascular injury to the tracts. When the resection is taken close to the tracts, there is greater risk of mechanical injury to the tracts themselves. In cases in which the resection is stopped more distant from the functional pathways, inadvertent injury to the perforating arteries supplying portions of the motor fibers may result in significant morbidity. In two select patients who experienced long-term deficits, volumes of the resection cavity (pink) and regions of restricted DWI (green) on postoperative scans were segmented and overlapped with the preoperative motor fibers modeled by DMRI-FT (blue), to imply possible tract injury due to mechanical and/or ischemic injury. In the first of these illustrative cases (Fig. 1A-D), the patient suffered a long-term deficit, but the subcortical fibers were never identified by stimulation. Interestingly, the DMRI-FT CST did not intersect with the resection cavity, but a large bundle of motor fibers were seen to be located within the region of postoperative restricted DWI. In contrast, in the second case (Fig. 1E-H), intraoperative stimulation did identify the motor tracts, and the patient experienced a persistent motor deficit. As expected, a significant portion of the descending motor fibers can be observed running through the resection cavity (pink).

\section{Conclusions}

Resection of tumors within or adjacent to the motor cortex or subcortical tracts can be challenging and is associated with a high rate of immediate postoperative neurological morbidity. In our experience, $30 \%$ of patients had a new or worsened motor deficit postoperatively; however, most of these deficits were transient. When the resection was carried close to the motor fibers and direct stimulation localized the descending fibers, the rate of long-term 
TABLE 5. Diffusion-weighted imaging abnormalities on postoperative imaging among patients with deficits

\begin{tabular}{lccc}
\hline Parameter & $\begin{array}{c}\text { No. of Patients } \\
\text { w/ Long-Term } \\
\text { Deficits }\end{array}$ & $\begin{array}{c}\text { No. of Patients w/ } \\
\text { Significant DWI } \\
\text { Restricted Area }\end{array}$ & $\begin{array}{c}p \\
\text { Value }\end{array}$ \\
\hline All patients & 49 & $20(41 \%)$ & \\
\hline Subcortical pathway & & $11(31 \%)$ & \\
\hline Found & 36 & $9(69 \%)$ & \\
\hline Not found & 13 & & 0.049 \\
\hline Histology & & $17(46 \%)$ & \\
\hline High grade & 37 & $3(25 \%)$ & \\
\hline Low grade & 12 & & 0.75 \\
\hline Preop deficit & & $5(36 \%)$ & \\
\hline Yes & 14 & $15(43 \%)$ & \\
\hline No & 35 &
\end{tabular}

motor deficit was $12 \%$ (7.7\% mild, $4.3 \%$ moderate/severe). If the subcortical pathways could not be identified, the incidence of long-term motor morbidity was $3.2 \%(1.7 \%$ mild, $1.4 \%$ moderate/severe). Using stimulation mapping techniques to identify descending motor pathways during resection of tumors in perirolandic regions facilitates the reduction of neurological morbidity to acceptable rates in these high-risk cases. Among the subcohort of patients who experienced a persistent motor deficit and in whom the subcortical pathway could not be localized by stimulation, there was a high incidence of restricted diffusion on postoperative imaging, suggesting that vascular injury to the tracts may be a major contributor to permanent deficits. Further study is needed to directly test the relationship between restricted diffusion and postoperative morbidity, but our results suggest that particular care should be taken to avoid injuring or coagulating perforating vessels while working near subcortical motor pathways.

\section{Acknowledgments}

We would like to acknowledge Jing Li for her assistance querying the local database to identify the relevant patient population included in this study.

\section{References}

1. Bello L, Castellano A, Fava E, Casaceli G, Riva M, Scotti $\mathrm{G}$, et al: Intraoperative use of diffusion tensor imaging fiber tractography and subcortical mapping for resection of gliomas: technical considerations. Neurosurg Focus 28(2):E6, 2010

2. Bello L, Gambini A, Castellano A, Carrabba G, Acerbi F, Fava E, et al: Motor and language DTI Fiber Tracking combined with intraoperative subcortical mapping for surgical removal of gliomas. Neuroimage 39:369-382, 2008

3. Bello L, Riva M, Fava E, Ferpozzi V, Castellano A, Raneri F, et al: Tailoring neurophysiological strategies with clinical context enhances resection and safety and expands indications in gliomas involving motor pathways. Neuro Oncol 16:1110-1128, 2014

4. Berger MS: Lesions in functional ("eloquent") cortex and subcortical white matter. Clin Neurosurg 41:444-463, 1994

5. Berman JI, Berger MS, Chung SW, Nagarajan SS, Henry RG:
Accuracy of diffusion tensor magnetic resonance imaging tractography assessed using intraoperative subcortical stimulation mapping and magnetic source imaging. J Neurosurg 107:488-494, 2007

6. Bucci M, Mandelli ML, Berman JI, Amirbekian B, Nguyen C, Berger MS, et al: Quantifying diffusion MRI tractography of the corticospinal tract in brain tumors with deterministic and probabilistic methods. Neuroimage Clin 3:361-368, 2013

7. Carrabba G, Fava E, Giussani C, Acerbi F, Portaluri F, Songa $\mathrm{V}$, et al: Cortical and subcortical motor mapping in rolandic and perirolandic glioma surgery: impact on postoperative morbidity and extent of resection. J Neurosurg Sci 51:45-51, 2007

8. Castellano A, Bello L, Michelozzi C, Gallucci M, Fava E, Iadanza A, et al: Role of diffusion tensor magnetic resonance tractography in predicting the extent of resection in glioma surgery. Neuro Oncol 14:192-202, 2012

9. Chamberland M, Whittingstall K, Fortin D, Mathieu D, Descoteaux M: Real-time multi-peak tractography for instantaneous connectivity display. Front Neuroinform 8:59, 2014

10. De Witt Hamer PC, Robles SG, Zwinderman AH, Duffau H, Berger MS: Impact of intraoperative stimulation brain mapping on glioma surgery outcome: a meta-analysis. J Clin Oncol 30:2559-2565, 2012

11. Duffau H: Intraoperative direct subcortical stimulation for identification of the internal capsule, combined with an image-guided stereotactic system during surgery for basal ganglia lesions. Surg Neurol 53:250-254, 2000

12. Duffau H, Capelle L, Denvil D, Sichez N, Gatignol P, Taillandier L, et al: Usefulness of intraoperative electrical subcortical mapping during surgery for low-grade gliomas located within eloquent brain regions: functional results in a consecutive series of 103 patients. J Neurosurg 98:764-778, 2003

13. Hervey-Jumper SL, Li J, Lau D, Molinaro AM, Perry DW, Meng L, et al: Awake craniotomy to maximize glioma resection: methods and technical nuances over a 27 -year period. J Neurosurg 123:325-339, 2015

14. Jenkinson M, Bannister P, Brady M, Smith S: Improved optimization for the robust and accurate linear registration and motion correction of brain images. Neuroimage 17:825-841, 2002

15. Jenkinson $\mathrm{M}$, Smith $\mathrm{S}$ : A global optimisation method for robust affine registration of brain images. Med Image Anal 5:143-156, 2001

16. Kamada K, Todo T, Ota T, Ino K, Masutani Y, Aoki S, et al: The motor-evoked potential threshold evaluated by tractography and electrical stimulation. J Neurosurg 111:785-795, 2009

17. Keles GE, Lundin DA, Lamborn KR, Chang EF, Ojemann G, Berger MS: Intraoperative subcortical stimulation mapping for hemispherical perirolandic gliomas located within or adjacent to the descending motor pathways: evaluation of morbidity and assessment of functional outcome in 294 patients. J Neurosurg 100:369-375, 2004

18. Mandelli ML, Berger MS, Bucci M, Berman JI, Amirbekian B, Henry RG: Quantifying accuracy and precision of diffusion MR tractography of the corticospinal tract in brain tumors. J Neurosurg 121:349-358, 2014

19. Mikuni N, Okada T, Enatsu R, Miki Y, Hanakawa T, Urayama $\mathrm{S}$, et al: Clinical impact of integrated functional neuronavigation and subcortical electrical stimulation to preserve motor function during resection of brain tumors. J Neurosurg 106:593-598, 2007

20. Nimsky C, Ganslandt O, Hastreiter P, Wang R, Benner T, Sorensen AG, et al: Intraoperative diffusion-tensor MR imaging: shifting of white matter tracts during neurosurgical procedures-initial experience. Radiology 234:218-225, 2005 
21. Nossek E, Korn A, Shahar T, Kanner AA, Yaffe H, Marcovici $\mathrm{D}$, et al: Intraoperative mapping and monitoring of the corticospinal tracts with neurophysiological assessment and 3 -dimensional ultrasonography-based navigation. Clinical article. J Neurosurg 114:738-746, 2011

22. Ozawa N, Muragaki Y, Nakamura R, Iseki H: Identification of the pyramidal tract by neuronavigation based on intraoperative diffusion-weighted imaging combined with subcortical stimulation. Stereotact Funct Neurosurg 87:18-24, 2009

23. Plans G, Fernández-Conejero I, Rifà-Ros X, FernándezCoello A, Rosselló A, Gabarrós A: Evaluation of the highfrequency monopolar stimulation technique for mapping and monitoring the corticospinal tract in patients with supratentorial gliomas. A proposal for intraoperative management based on neurophysiological data analysis in a series of 92 patients. Neurosurgery 81:585-594, 2017

24. Prabhu SS, Gasco J, Tummala S, Weinberg JS, Rao G: Intraoperative magnetic resonance imaging-guided tractography with integrated monopolar subcortical functional mapping for resection of brain tumors. Clinical article. J Neurosurg 114:719-726, 2011

25. Raabe A, Beck J, Schucht P, Seidel K: Continuous dynamic mapping of the corticospinal tract during surgery of motor eloquent brain tumors: evaluation of a new method. J Neurosurg 120:1015-1024, 2014

26. Raco A, Pesce A, Fraschetti F, Frati A, D'Andrea G, Cimatti $\mathrm{M}$, et al: Motor outcomes after surgical resection of lesions involving the motor pathway: a prognostic evaluation scale. World Neurosurg 103:748-756, 2017

27. Sala F, Lanteri P: Brain surgery in motor areas: the invaluable assistance of intraoperative neurophysiological monitoring. J Neurosurg Sci 47:79-88, 2003

28. Seidel K, Beck J, Stieglitz L, Schucht P, Raabe A: The warning-sign hierarchy between quantitative subcortical motor mapping and continuous motor evoked potential monitoring during resection of supratentorial brain tumors. J Neurosurg 118:287-296, 2013

29. Tarapore PE, Tate MC, Findlay AM, Honma SM, Mizuiri D, Berger MS, et al: Preoperative multimodal motor mapping: a comparison of magnetoencephalography imaging, navigated transcranial magnetic stimulation, and direct cortical stimulation. J Neurosurg 117:354-362, 2012

30. Trinh VT, Fahim DK, Shah K, Tummala S, McCutcheon IE, Sawaya R, et al: Subcortical injury is an independent predictor of worsening neurological deficits following awake craniotomy procedures. Neurosurgery 72:160-169, 2013

31. Vassal F, Schneider F, Nuti C: Intraoperative use of diffusion tensor imaging-based tractography for resection of gliomas located near the pyramidal tract: comparison with subcortical stimulation mapping and contribution to surgical outcomes. Br J Neurosurg 27:668-675, 2013
32. Wakana S, Caprihan A, Panzenboeck MM, Fallon JH, Perry M, Gollub RL, et al: Reproducibility of quantitative tractography methods applied to cerebral white matter. Neuroimage 36:630-644, 2007

33. Wang JY, Abdi H, Bakhadirov K, Diaz-Arrastia R, Devous MD Sr: A comprehensive reliability assessment of quantitative diffusion tensor tractography. Neuroimage 60:11271138,2012

34. Wu JS, Zhou LF, Tang WJ, Mao Y, Hu J, Song YY, et al: Clinical evaluation and follow-up outcome of diffusion tensor imaging-based functional neuronavigation: a prospective, controlled study in patients with gliomas involving pyramidal tracts. Neurosurgery 61:935-949, 2007

35. Yingling CD, Ojemann S, Dodson B, Harrington MJ, Berger MS: Identification of motor pathways during tumor surgery facilitated by multichannel electromyographic recording. J Neurosurg 91:922-927, 1999

36. Zhu FP, Wu JS, Song YY, Yao CJ, Zhuang DX, Xu G, et al: Clinical application of motor pathway mapping using diffusion tensor imaging tractography and intraoperative direct subcortical stimulation in cerebral glioma surgery: a prospective cohort study. Neurosurgery 71:1170-1184, 2012

\section{Disclosures}

The authors report no conflict of interest concerning the materials or methods used in this study or the findings specified in this paper.

\section{Author Contributions}

Conception and design: Berger, Han. Acquisition of data: Han, Morshed, Troncon. Analysis and interpretation of data: Berger, Han, Troncon, Jordan, Henry. Drafting the article: Han, Morshed. Critically revising the article: Berger, Morshed, Jordan, HerveyJumper. Reviewed submitted version of manuscript: Berger, Han, Morshed, Jordan, Henry, Hervey-Jumper. Statistical analysis: Han. Study supervision: Berger, Han, Henry, Hervey-Jumper.

\section{Supplemental Information \\ Previous Presentations}

The content of this paper was presented at the 22nd Annual Scientific Meeting and Education Day of the Society for NeuroOncology held in San Francisco, CA, on November 16-19, 2017.

\section{Correspondence}

Mitchel S. Berger: University of California, San Francisco, CA. mitchel.berger@ucsf.edu. 